\title{
Phenolic Compound Profiles in Grape Skins of Cabernet Sauvignon, Merlot, Syrah and Marselan Cultivated in the Shacheng Area (China)
}

\author{
P.B. Shi ${ }^{1,2, \#, ~ T . X . ~ Y u e ~}{ }^{1,3, \#, ~ L . L . ~ A i ~}{ }^{1}$, Y.F. Cheng ${ }^{1,3}$, J.F. Meng ${ }^{1}$, M.H. Li ${ }^{4}$, Z.W. Zhang ${ }^{1,5^{*}}$ \\ (1) College of Enology, Northwest A\&F University, Yangling, Shaanxi, 712100, China \\ (2) Hebei Normal University of Science \& Technology, Qinhuangdao, Hebei, 066600, China \\ (3) Baotou Light Industry Vocational Technical College, Baotou, Neimenggu, 014035, China \\ (4) Planning and Construction Service Center of the People's Government of Beigu, Beigu, Yunnan, 652112, China \\ (5) Shaanxi Engineering Research Center for Viti-Viniculture, Northwest A\&F University, Yangling, Shaanxi, 712100, China
}

Submitted for publication: March 2016

Accepted for publication: May 2016

Key words: Phenolic compounds, anthocyanins, wine grape, HPLC-MS, cluster analysis

\begin{abstract}
The phenolic compounds in the grape skins of Cabernet Sauvignon (CS), Merlot (ML), Syrah (SY) and Marselan (MS) from Shacheng, in China, were compared using HPLC-MS/MS. The results showed that the types and levels of phenolic compounds varied greatly with cultivars. Malvidin derivatives were the main anthocyanins. CS and ML showed a higher content of malvidin-3-O-(6-O-acetyl)-glucoside than malvidin-3-O-(trans-6- $O$ - coumaryl)-glucoside, while SY and MS differed from CS and ML. ML had higher delphinidin and cyanidin derivatives, SY had higher peonidin derivatives, while malvidin and petunidin were higher in MS. The total content of flavonols, flavan-3-ols, phenolic acids and stilbenes in grape skins showed no difference among CS, ML and MS. Isorhamnetin-3-O-glucoside (CS, ML, MY), quercetin-3$O$-glucoside (SY), procyanidin trimer (SY, MS), procyanidin dimer (CS, ML), syringetin-3-O-glucoside, trans-cinnamic acid and resveratrol were the most abundant non-anthocyanin phenolic compounds. Cluster analysis showed that CS and ML, and SY and MS had similar phenolic profiles.
\end{abstract}

\section{INTRODUCTION}

Phenolic compounds in red grape skins are one of the most important parameters in determining red wine character and quality, and directly influence consumers' overall acceptance (Li et al., 2011). They contribute to the mouthfeel, colour and stability of red wines; some of them also exhibit potent biological activities (Gómez-Alonso et al., 2007). Phenolic compounds, mostly originating from grape berries, are transferred into wine during the winemaking process (Salas et al., 2003). The main phenolic compounds in red grapes and red wine are anthocyanins and non-anthocyanins, which include flavonols, flavan-3-ols, phenolic acids (including hydroxycinnamic acids and hydroxybenzoic acids) and stilbenes (Monagas et al., 2005; Li et al., 2011; Zhang et al., 2015). Anthocyanins, which are directly responsible for red grape and red wine colour (Mateus et al., 2002; Revilla et al., 2009), are composed of the monoglucosides of five anthocyanidins, namely delphinidin, cyanidin, petunidin, peonidin and malvidin, along with the corresponding acetyl, p-coumaroyl and caffeoyl derivatives (Liang et al., 2008; Raúl et al., 2009). Flavonols, which influence red wine coloration by co-pigmentation (Boulton, 2001), consist of the glycosides of myricetin, quercetin, kaempferol, isorhamnetin, syringetin and laricitrin (Raúl et al., 2009). The flavan-3-ols found in the skin and seed are mainly catechin, epicatechin, gallocatechin, epigallocatechin and their corresponding polymers. Flavan-3-ols are mainly responsible for the astringency, bitterness and structure of wines (Monagas et al., 2005), and they also play an important role in the stabilization of the red colour in wines (Sun et al., 2007). Stilbenes (mainly resveratrol) exhibit significant antioxidant properties in the prevention of arteriosclerosis and coronary heart disease (Sun et al., 2002).

The amount of phenolic compounds in grapes is influenced by the grape variety, along with viticultural and environmental factors (which are usually described by the French term "terroir"), which include soil type, geographical location and weather conditions (Douglas et al., 2001; Brescia et al., 2002; Xing et al., 2015). Temperature plays a direct and important role in the formation of phenolic compounds; temperatures higher than $30^{\circ} \mathrm{C}$ are not conducive to anthocyanin synthesis (Spayd et al., 2002; Tarara et al., 2008). Lower night temperatures can result in greater accumulation of anthocyanins (Mori et al., 2005). Several studies have shown a positive association between

*Corresponding author: E-mail address: zhangzhw60@nwsuaf.edu.cn

\#These authors contributed equally to this work

Acknowledgements: The authors would like to express their gratitude to the National Technology System for Grape Industry (CARS-30-zp-9) 
sunlight exposure and flavone accumulation (Downey et al., 2004). Li et al. (2011) reported that "terroir" characteristics affect the flavonoid biosynthesis in grape berries, eventually leading to the difference in the phenol profile of wines from different regions.

Shacheng region is a traditional vine-growing region in the Hebei province of China. It is located in the Sangyang basin, which is a warm, semiarid region. The mean annual temperature is $12.5^{\circ} \mathrm{C}$. Active accumulated temperature $\left(\geq 10^{\circ} \mathrm{C}\right)$ is more than $3500^{\circ} \mathrm{C}$. Annual rainfall is $400 \mathrm{~mm}$. Solar radiation is high $\left(146.36 \mathrm{kcal} / \mathrm{cm}^{2}\right)$, and the annual frostless period is 160 days. The area under wine grapes in Shacheng region is 8300 ha, and the main cultivars are Cabernet Sauvignon, Merlot, Syrah, Marselan, etc. However, there is little information available on the phenolic compounds of wine grapes in the Shacheng region.

The purpose of this study was to investigate the phenolic compounds of the main wine grapes in the Shacheng region to help improve the quality of the grape berries by appropriate cultivation techniques, and to evaluate the winemaking potential of the different grape cultivars.

\section{MATERIALS AND METHODS \\ Materials}

The study was carried out at Donghuayuan town, which is located in the Shacheng region of Hebei province, China. The soil type is clay and sandy. The climate, which is influenced by abundant sunshine, moderate heat, a high temperature difference between day and night and cool weather in the summer, provides a good environment for vine growing.

Four red Vitis vinifera grapes, of the varieties Cabernet Sauvignon (CS), Merlot (ML), Syrah (SY) and Marselan (MS), were collected in 2010. The grapevines of each cultivar were four years old, planted at a spacing of $2.5 \mathrm{~m}$ $\times 1.0 \mathrm{~m}$ (row, vine). The grape berries were harvested at technological ripeness, depending on the sugar and acid content. The fresh grape samples were placed in freezer bags and taken to the laboratory immediately. Grape skins were peeled from the berries and frozen in liquid nitrogen, followed by grinding and lyophilisation. The grape skin powder was stored at $-40^{\circ} \mathrm{C}$ until used (Jin et al., 2009).

\section{Chemicals and standards}

Malvidin-3-O-glucoside standard was purchased from Extrasynthese SA (Genay, France). Quercetin, (+)-catechin, gallic acid, caffeic acid and trans-resveratrol standards were all purchased from Sigma Company (St. Louis, MI, USA). Ethyl acetate (analytical grade) was obtained from Xian Chemical Reagent Plant (Xian, China). HPLC-grade methanol, formic acid, acetic acid and acetonitrile were obtained from Fisher Company (Fairlawn, NJ, USA).

\section{Extraction and analysis of anthocyanins}

Grape skin powder $(0.50 \mathrm{~g})$ was immersed in methanol (10 $\mathrm{mL}$ ) containing $2 \%$ formic acid. The extraction was performed for $10 \mathrm{~min}$ with the assistance of ultrasonic vibration, and then the mixture was shaken in the dark at a rate of $150 \mathrm{rpm}$ for $30 \mathrm{~min}$ at $25^{\circ} \mathrm{C}$. The extract was centrifuged at $8000 \mathrm{~g}$ for $10 \mathrm{~min}$ and the supernatant was collected. The residues were extracted another three times.
All supernatants were mixed and evaporated to dryness using a rotary evaporator. Then the dry residual was re-dissolved in $10 \mathrm{~mL}$ mobile phase $(\mathrm{A}: \mathrm{B}=9: 1)(\mathrm{A}$ : aqueous $2 \%$ (vol) formic acid, B: acetonitrile containing 2\% (vol) formic acid). The final samples were filtered through $0.45 \mu \mathrm{m}$ filters (cellulose acetate and nitrocellulose, CAN) prior to HPLCMS analysis. All extractions were done in triplicate.

An Agilent 1100 series LC-MSD trap VL instrument equipped with a diode array detector and reverse phase column (Kromasil C18, $250 \mathrm{~mm} \times 4.6 \mathrm{~mm}, 5 \mu \mathrm{m}$ ) was used for qualitative and quantitative analyses of anthocyanin in the extracts. Elutions included two solutions: (A) aqueous $2 \%$ (vol) formic acid and (B) acetonitrile containing 2\% (vol) formic acid. The gradient was as follows: $4 \mathrm{~min}, 6 \%$ to $10 \% \mathrm{~B} ; 8 \mathrm{~min}, 10 \%$ to $25 \% \mathrm{~B} ; 1 \mathrm{~min}$, isocratic $25 \% \mathrm{~B} ; 7 \mathrm{~min}$, $25 \%$ to $40 \% \mathrm{~B} ; 15 \mathrm{~min}, 40 \%$ to $60 \% \mathrm{~B} ; 5 \mathrm{~min}, 60 \%$ to $100 \% \mathrm{~B}$; then $5 \mathrm{~min}, 100 \%$ to $6 \% \mathrm{~B}$. The flow rate was $1.0 \mathrm{~mL} / \mathrm{min}$. Injection volumes were $30 \mu \mathrm{L}$ and the detection wavelength was $525 \mathrm{~nm}$. MS conditions were as follows: Electrospray ionisation (ESI) interface, positive ion mode, $35 \mathrm{psi}$ nebuliser pressure, $10 \mathrm{~mL} / \mathrm{min}$ dry gas flow rate, $350^{\circ} \mathrm{C}$ dry gas temperature and scans at $\mathrm{m} / \mathrm{z} 100$ to 1000 (Jin et al., 2009). All analyses were performed in duplicate.

\section{Extraction and analysis of non-anthocyanins}

Grape skin powder $(2.00 \mathrm{~g})$ was macerated with distilled water $(5 \mathrm{~mL})$ and ethyl acetate $(45 \mathrm{~mL})$, and shaken in the dark for $30 \mathrm{~min}$. The supernatant was collected and the residue was extracted four times. All of the supernatants were mixed together and evaporated to dryness using a rotary evaporator, and then re-dissolved in methanol $(2 \mathrm{~mL})$. All extractions were done in triplicate. The determination and quantification of non-anthocyanins were carried out using HPLC-MS/MS, as described by Jin et al. (2009). Each sample was first filtered through a $0.22 \mu \mathrm{m}$ organic membrane and then injected into an Agilent 1200 series HPLC-MSD trap VL instrument, equipped with a variable wavelength detector and a reverse phase column (Zorbax SB-C18 column $3 \mathrm{~mm} \times 50 \mathrm{~mm}, 1.8 \mu \mathrm{m})$. Samples were eluted at a gradient using two mobile phases: (A) aqueous $1 \%$ acetic acid and (B) acetonitrile containing $1 \%$ acetic acid. The gradient was $5 \mathrm{~min}, 5 \%$ to $8 \% \mathrm{~B} ; 2 \mathrm{~min}, 8 \%$ to $12 \% \mathrm{~B}$; $5 \mathrm{~min}, 12 \%$ to $18 \% \mathrm{~B} ; 5 \mathrm{~min}, 18 \%$ to $22 \% \mathrm{~B} ; 2 \mathrm{~min}, 22 \%$ to $35 \% \mathrm{~B} ; 2 \mathrm{~min}, 35 \%$ to $100 \% \mathrm{~B} ; 4 \mathrm{~min}, 100 \% \mathrm{~B}$, then $2 \mathrm{~min}$, $100 \%$ to $5 \% \mathrm{~B}$, at a flow rate of $1.0 \mathrm{~mL} / \mathrm{min}$. The detection wavelength was $280 \mathrm{~nm}$ and the injection volume was $2 \mu \mathrm{L}$. The column temperature was $25^{\circ} \mathrm{C}$. The MS conditions were as follows: ESI, negative ion mode; nebuliser, 35 psi; dry gas flow, $10 \mathrm{~mL} / \mathrm{min}$; dry gas temperature, $325^{\circ} \mathrm{C}$; scan, $100 \mathrm{~m} / \mathrm{z}$ to $1000 \mathrm{~m} / z$. All analyses were done in duplicate.

\section{Quantification of phenolic compounds}

Anthocyanins, flavonol, flavan-3-ols, hydroxybenzoic acid, hydroxycinnamic acids and stilbenes were calculated by using an external standard calibration curve for each compound, and were expressed as $\mathrm{mg}$ of malvidin-3-Oglucoside $(\mathrm{ME})$, quercetin, $(\mathrm{QE}),(+)$-catechin $(\mathrm{CE})$, gallic acid (GAE), caffeic acid (CAE) and trans-resveratrol (RE) equivalents per $\mathrm{kg}$ of dry grape skin. 


\section{Statistical analysis}

SPSS 16.0 for Windows was used for the variance and cluster analysis. Data were expressed as mean \pm standard deviation of three independent experiments with two replicates.

\section{RESULTS AND DISCUSSION}

\section{The anthocyanin composition of grape skin}

As shown in Fig. 1, the total content of anthocyanin varied from $10847.02 \mathrm{mg} \mathrm{ME} / \mathrm{kg}$ (ML) to $20790.75 \mathrm{mg} \mathrm{ME} / \mathrm{kg}$ (MS). There was a significant difference in the anthocyanin contents in the grape skins of the four varieties, with a decreasing order: MS $>\mathrm{SY}>\mathrm{CS}>\mathrm{ML}$. It has been reported that there are no significant differences in the anthocyanin contents of Cabernet Sauvignon and Merlot (Jensen et al., 2008), which is inconsistent with our results. In the grape cultivars studied, malvidin and its derivatives (46.33\% to $71.76 \%$ of total anthocyanins) are the main anthocyanins present in the skin. Compared with other cultivars, ML had higher delphinidin, cyanidin and their derivatives; SY had higher peonidin and its derivatives; and MS had higher malvidin and petunidin and their derivatives.

A total of 21 different anthocyanins were identified from four grape cultivars using HPLC-ESI-MS/MS (Table 1), including five glucosides, five acetyl glucosides, seven coumaroyl glucosides, two caffeoyl glucosides and two polymeric anthocyanins, all of which could be classified into the monoglucosides and derivatives of five anthocyanidins: delphinidin, cyanidin, petunidin, peonidin and malvidin. As in previous studies (Revilla et al., 2001; Kallithraka et al., 2005; Raúl et al., 2009), malvidin-3-O-glucoside was the most abundant anthocyanin in CS, ML, SY and MS, accounting for $36.51 \%, 28.64 \%, 26.52 \%$ and $38.95 \%$ of the total anthocyanin content respectively. In addition to this compound, delphinidin-3-O-glucoside (CS, ML, MS) and peonidin-3-O-glucoside (SY) were the second most abundant non-acylated anthocyanins. It is worth mentioning that malvidin-3-O-glucoside-pyruvic acid was only detected in SY, and malvidin-3-(6-O-caffeoyl)-glucoside was not detected in ML and MS. Mazza et al. (1999) reported that malvidin-3-O- (6-O-acetyl)-glucoside, along with malvidin3-O-(6-O-coumaryl)-glucoside, was one of the most important derivatives for the characterisation of varieties. In our study, CS and ML showed a high content of malvidin3-O-(6-O-acetyl)-glucoside, followed by malvidin-3-O(trans-6-O-coumaryl)-glucoside, as in a previous study (Revilla et al., 2001). However, SY and MS had higher contents of malvidin-3-O-(trans-6-O-coumaryl)-glucoside (SY: $4324.94 \mathrm{mg} \mathrm{ME} / \mathrm{kg}, 23.73 \%$ of total anthocyanins; MS: $3596.14 \mathrm{mg} \mathrm{ME} / \mathrm{kg}, 17.30 \%$ of total anthocyanins) than malvidin-3-O-(6-O-acetyl)-glucoside (SY: $2058.55 \mathrm{mg}$ $\mathrm{ME} / \mathrm{kg}, 11.30 \%$ of total anthocyanins; MS: $3127.17 \mathrm{mg}$ $\mathrm{ME} / \mathrm{kg}, 15.04 \%$ of total anthocyanins).

\section{The non-anthocyanin composition of grape skin}

The non-anthocyanin composition of the CS, ML, SY and MS grape skins obtained from Donghuayuan is summarised in Table 2. A total of 25 non-anthocyanin phenolic compounds were identified and quantified in these samples, including 14 flavonols, four flavan-3-ols, four hydroxybenzoic acids, two hydroxycinnamic acids and one stilbene.

\section{The flavonol composition}

Flavonols exist mainly as the four main aglycones: myricetin, quercetin, kaempferol and isorhamnetin (Monagas et al., 2005). As shown in Table 2, 14 flavonol compounds in the grape skins varied significantly among the grape cultivars studied. Myricetin-3-O-glucuronide was detected only in ML, but no myricetin-3-O-galactoside was detected in ML.

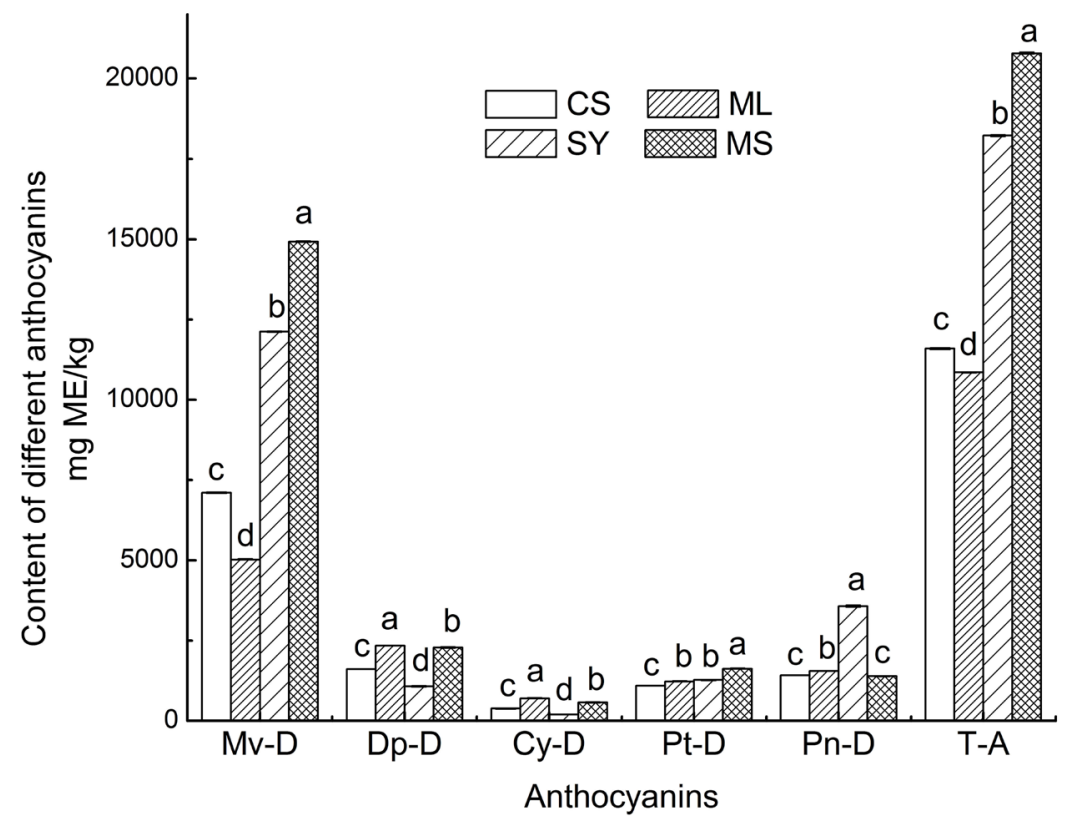

FIGURE 1

The content of different anthocyanin derivatives of four grape cultivars. ME: malvidin-3-O-glucoside; Mv-D: malvidin and its derivatives; Dp-D: delphinidin and its derivatives; Cy-D: cyanidin and its derivatives; Pt-D: petunidin and its derivatives; Pn-D: peonidin and its derivatives; T-A: total anthocyanins. Different small letters indicate a significant difference at $\mathrm{P}<0.05$. 
TABLE 1

The content of anthocyanin compounds in the berry skins of four grape cultivars.

\begin{tabular}{|c|c|c|c|c|c|}
\hline \multirow[b]{2}{*}{ Anthocyanins } & \multirow{2}{*}{$\begin{array}{c}{\left[\mathrm{M}^{+}\right] \text {(Frag. }} \\
\left.\mathrm{MS}^{2} \mathrm{~m} / z\right)\end{array}$} & \multicolumn{4}{|c|}{ Content (mg ME/kg) } \\
\hline & & $\mathrm{CS}$ & ML & SY & MS \\
\hline Delphinidin-3-O-glucoside & $465(303)$ & $1307.52 \pm 13.32$ & $2028.26 \pm 8.64$ & $745.14 \pm 8.93$ & $1355.59 \pm 6.92$ \\
\hline Cyanidin-3-O-glucoside & $449(287)$ & $239.48 \pm 5.69$ & $541.06 \pm 5.15$ & $146.53 \pm 3.27$ & $144.01 \pm 4.88$ \\
\hline Petunidin-3-O-glucoside & $479(317)$ & $792.60 \pm 6.12$ & $830.02 \pm 6.32$ & $970.85 \pm 7.92$ & $1198.82 \pm 5.63$ \\
\hline Peonidin-3-O-glucoside & $463(301)$ & $824.27 \pm 5.23$ & $239.54 \pm 2.11$ & $1105.45 \pm 8.68$ & $691.62 \pm 4.32$ \\
\hline Malvidin-3-O-glucoside & $493(331)$ & $4231.81 \pm 6.24$ & $3107.13 \pm 8.93$ & $4833.27 \pm 10.67$ & $8098.94 \pm 7.89$ \\
\hline Delphinidin-3-O-(6-O-acetyl)-glucoside & $507(303)$ & $297.21 \pm 7.32$ & $232.81 \pm 4.58$ & $157.23 \pm 3.43$ & $354.80 \pm 5.44$ \\
\hline Peonidin-3-O-glucoside-pyruvic acid & $531(301)$ & $35.90 \pm 3.45$ & $42.05 \pm 2.46$ & $53.51 \pm 5.32$ & $60.46 \pm 3.87$ \\
\hline Malvidin-3-O-glucoside-pyruvic acid & $603(399)$ & nd & nd & $26.39 \pm 2.29$ & nd \\
\hline Cyanidin-3-O-(6-O-acetyl)-glucoside & $491(287)$ & $79.54 \pm 4.61$ & $118.89 \pm 3.38$ & $46.34 \pm 3.59$ & $68.67 \pm 5.44$ \\
\hline Petunidin-3-O-(6-O-acetyl)-glucoside & $521(317)$ & $270.28 \pm 5.38$ & $247.98 \pm 4.32$ & $265.46 \pm 3.15$ & $394.64 \pm 1.59$ \\
\hline Delphinidin-3-O-(6-O-coumaryl)-glucoside & $611(303)$ & $\operatorname{tr}$ & $81.93 \pm 2.78$ & $163.37 \pm 2.98$ & $571.25 \pm 4.44$ \\
\hline Peonidin-3-O-(6-O-acetyl)-glucoside & $505(301)$ & $329.68 \pm 6.24$ & $576.65 \pm 3.84$ & $835.08 \pm 4.69$ & $48.31 \pm 3.61$ \\
\hline Malvidin-3-O-(6-O-acetyl)-glucoside & $535(331)$ & $2023.29 \pm 8.98$ & $1079.31 \pm 7.43$ & $2058.55 \pm 6.33$ & $3127.17 \pm 7.65$ \\
\hline Peonidin-3-O-(6-O-caffeoyl)-glucoside & $625(301)$ & $27.31 \pm 4.21$ & $151.85 \pm 2.74$ & $233.00 \pm 2.15$ & $163.46 \pm 3.33$ \\
\hline Cyanidin-3-O-(6-O-coumaryl)-glucoside & $595(287)$ & $61.88 \pm 5.33$ & $37.46 \pm 3.24$ & $\operatorname{tr}$ & $361.98 \pm 5.68$ \\
\hline Malvidin-3-(6-O-caffeoyl)-glucoside & $655(331)$ & $79.45 \pm 4.27$ & nd & $622.91 \pm 5.68$ & nd \\
\hline Petunidin-3-O-(6-O-coumaryl)-glucoside & $625(317)$ & $25.89 \pm 3.16$ & $150.44 \pm 2.11$ & $35.98 \pm 3.38$ & $31.79 \pm 2.92$ \\
\hline Peonidin-3-O-(cis-6-O-coumaryl)- glucoside & $609(301)$ & $25.89 \pm 2.58$ & $41.53 \pm 1.58$ & $56.52 \pm 2.73$ & $44.18 \pm 3.47$ \\
\hline Malvidin-3-O-(cis-6-O-coumaryl)-glucoside & $639(331)$ & $52.64 \pm 4.32$ & $55.38 \pm 3.23$ & $280.37 \pm 3.11$ & $97.55 \pm 4.32$ \\
\hline Peonidin-3-O-(trans-6-O-coumaryl)-glucoside & $609(301)$ & $169.90 \pm 5.84$ & $501.63 \pm 4.32$ & $1260.80 \pm 6.32$ & $381.36 \pm 7.88$ \\
\hline Malvidin-3-O-(trans-6-O-coumaryl)-glucoside & $639(331)$ & $715.19 \pm 6.11$ & $783.10 \pm 4.88$ & $4324.94 \pm 5.66$ & $3596.14 \pm 6.52$ \\
\hline
\end{tabular}

Values are means of duplicate determination \pm S.D. nd: not detected. tr: trace. ME: malvidin-3-O-glucoside

Kaempferol-3-O-galactoside was not detected in MS. Among the flavonols, isorhamnetin-3-O-glucoside was the most abundant in CS, ML and MS, but SY showed a higher level of quercetin-3-O-glucoside, consistent with Jin et al. (2009). Total flavonol contents ranged from $2285.5 \mathrm{mg} \mathrm{QE} / \mathrm{kg}$ (ML) to $2871.00 \mathrm{mg}$ QE/kg (SY), higher than previously described (Mattivi et al. 2006; Jin et al., 2009). The total flavonol content was higher than in other non-anthocyanins, accounting for from $82.18 \%$ (SY) to $86.14 \%$ (ML) of total non-anthocyanins content. Moreover, the total flavonol content of SY was significantly higher than that of ML $(P<0.05)$ (Fig. 2a).

\section{The flavan-3-ol composition}

Four flavan-3-ols detected in the grape skins of CS, ML, SY and MS are shown in Table 2. They are gallocatechin, procyanidin dimer, $(+)$-catechin and procyanidin trimer. No gallocatechin was found in SY and MS. Procyanidin trimer was the most abundant in SY and MS, while CS and ML had higher levels of procyanidin dimer and $(+)$-catechin respectively.

Total flavan-3-ol contents ranged from $156.79 \mathrm{mg} \mathrm{CE} / \mathrm{kg}$ (MS) to $200.59 \mathrm{mg} \mathrm{CE} / \mathrm{kg}$ (SY), accounting for $5.56 \%$ to $6.57 \%$ of total non-anthocyanin contents, a little higher than that recorded in the study of Jin et al. (2009), which was done at the foot of Qi-lian Mountain in northwest China. It appears that a warm climate is beneficial to producing a high content of flavan-3-ols (Rodriguez et al., 2006; Fernandez et al., 2007). Nevertheless, no significant $(P<0.05 ; P<0.01)$ differences were found among the grape cultivars studied (Fig. 2b).

\section{The phenolic acid and stilbene composition}

Six phenolic acids (four hydroxybenzoic acids, two hydroxycinnamic acids) and one stilbene were identified and quantified in the four wine grape cultivars (Table 2). The hexose ester of protocatechuic acid was not detected in CS, and no dimer (epi)gallocatechin-(epi)catechin was detected in MS. Syringetin-3-O-glucoside was the most abundant hydroxybenzoic acid. Total hydroxybenzoic acid contents ranged from $88.44 \mathrm{mg} \mathrm{GAE} / \mathrm{kg}$ (CS) to $116.36 \mathrm{mg} \mathrm{GAE} / \mathrm{kg}$ (SY), and this was about $3.10 \%$ to $3.51 \%$ of the total nonanthocyanins. As observed for the flavan-3-ol concentration, the level of total hydroxybenzoic acids showed no significant $(P<0.05 ; P<0.01)$ differences among the grape cultivars studied (Fig. 2c).

trans-Cinnamic acid was the most abundant hydroxycinnamic acid among the grape cultivars studied. Total hydroxycinnamic acid contents ranged from $7.31 \mathrm{mg}$ $\mathrm{CAE} / \mathrm{kg}$ (CS) to $16.38 \mathrm{mg} \mathrm{CAE} / \mathrm{kg}$ (SY), which was about $0.26 \%$ to $0.49 \%$ of the total non-anthocyanins. SY had a higher content of hydroxycinnamic acids than CS, while the others had no significant differences (Fig. 2d).

Stilbenes are phytoalexins that are directly related to environmental stress. Resveratrol was identified and quantified in the CS, SY and MS. The content of resveratrol 
TABLE 2

The content of non-anthocyanin compounds in the berry skins of four grape cultivars $(\mathrm{mg} / \mathrm{kg})$.

\begin{tabular}{|c|c|c|c|c|c|}
\hline \multirow{2}{*}{$\begin{array}{l}\text { Non-anthocyanin } \\
\text { phenolic compounds }\end{array}$} & \multirow{2}{*}{$\begin{array}{l}(\mathrm{M}-\mathrm{H})^{-} \\
\left(\text {Frag. } \mathrm{MS}^{2} \mathrm{~m} / \mathrm{z}\right)\end{array}$} & \multicolumn{4}{|c|}{ Content } \\
\hline & & CS & ML & SY & MS \\
\hline \multicolumn{6}{|l|}{ Flavonols: } \\
\hline Quercetin-3-O-hexoside & $463(301)$ & $61.57 \pm 11.38$ & $39.88 \pm 0.15$ & $43.53 \pm 0.38$ & $50.71 \pm 1.50$ \\
\hline Myricetin-3-O-glucuronide & $477(301)$ & nd & $228.54 \pm 5.61$ & nd & nd \\
\hline Myricetin-3-O-galactoside & 479 (317) & $206.52 \pm 6.56$ & nd & $167.34 \pm 6.04$ & $221.29 \pm 0.03$ \\
\hline Isorhamnetin-3-O-galactoside & $477(315)$ & $64.83 \pm 8.75$ & $118.25 \pm 4.16$ & $47.36 \pm 2.73$ & $30.46 \pm 4.50$ \\
\hline Myricetin-3-O-glucoside & $479(317,179,151)$ & $233.86 \pm 5.96$ & $141.72 \pm 4.03$ & $200.97 \pm 17.42$ & $248.2 \pm 44.31$ \\
\hline Dihydroquercetin-3'-O-rhamnoside & $449(285,303)$ & $72.51 \pm 3.87$ & $122.87 \pm 22.75$ & $62.08 \pm 2.95$ & $71.42 \pm 18.12$ \\
\hline Quercetin-3-O-galactoside & $463(301)$ & $70.76 \pm 6.43$ & $94.17 \pm 12.32$ & $120.1 \pm 3.56$ & $71.75 \pm 0.01$ \\
\hline Quercetin-3-O-glucuronide & $477(301)$ & $128.41 \pm 18.40$ & $215.81 \pm 15.40$ & $213.67 \pm 4.43$ & $146.87 \pm 1.00$ \\
\hline Quercetin-3-O-glucoside & $463(301)$ & $245.65 \pm 34.76$ & $418.38 \pm 23.62$ & $772.01 \pm 18.70$ & $238.38 \pm 6.85$ \\
\hline Laricitrin-3-O-glucoside & $493(331)$ & $55.27 \pm 3.55$ & $62.2 \pm 24.56$ & $72.42 \pm 5.02$ & $50.07 \pm 0.38$ \\
\hline Kaempferol-3-O-galactoside & $447(285)$ & $56.74 \pm 6.05$ & $74.39 \pm 14.17$ & $55.6 \pm 3.58$ & nd \\
\hline Kaempferol-3-O-glucoside & $447(285)$ & $423.22 \pm 74.88$ & $281.95 \pm 59.50$ & $360.42 \pm 12.56$ & $371.54 \pm 0.08$ \\
\hline Isorhamnetin-3-O-glucoside & $477(315)$ & $584.27 \pm 77.89$ & $427.61 \pm 33.19$ & $698.67 \pm 77.72$ & $622.26 \pm 81.97$ \\
\hline Quercetin-3-O-rutinoside & $609(301)$ & $254.90 \pm 24.98$ & $59.8 \pm 0.04$ & $56.88 \pm 0.05$ & $300.20 \pm 2.03$ \\
\hline \multicolumn{6}{|l|}{ Flavan-3-ols: } \\
\hline Gallocatechin & $305(179,217,137,125)$ & $29.99 \pm 6.60$ & $29.49 \pm 2.06$ & nd & nd \\
\hline Procyanidin dimer & $577(425,289)$ & $64.38 \pm 7.42$ & $44.1 \pm 4.48$ & $42.66 \pm 4.96$ & $19.08 \pm 4.21$ \\
\hline$(+)$-Catechin & $289(245)$ & $41.3 \pm 12.86$ & $50.43 \pm 5.56$ & $63.8 \pm 0.80$ & $35.56 \pm 0.01$ \\
\hline Procyanidin trimer & $865(695,577,287)$ & $48.85 \pm 3.75$ & $50.23 \pm 2.35$ & $94.12 \pm 52.86$ & $102.15 \pm 6.79$ \\
\hline \multicolumn{6}{|l|}{ Hydroxybenzoic acids } \\
\hline Dimer (epi)gallocatechin-(epi)catechin & $593(425,289,407)$ & $1.29 \pm 0.59$ & $0.55 \pm 0.02$ & $0.14 \pm 0.01$ & nd \\
\hline Hexose ester of protocatechuic acid & $315(153)$ & nd & $10.69 \pm 1.53$ & $10.65 \pm 5.09$ & $\operatorname{tr}$ \\
\hline Hexose ester of vanillic acid & $329(191,167)$ & $25.55 \pm 7.03$ & $22.93 \pm 0.80$ & $29.05 \pm 2.07$ & $10.32 \pm 3.54$ \\
\hline Syringetin-3-O-glucoside & $507(345)$ & $61.60 \pm 10.90$ & $57.02 \pm 26.86$ & $76.52 \pm 45.13$ & $87.61 \pm 11.86$ \\
\hline \multicolumn{6}{|l|}{ Hydroxycinnamic acids: } \\
\hline Hexose ester of ferulic acid & $355(193)$ & $3.81 \pm 0.83$ & $2.98 \pm 0.08$ & $3.83 \pm 0.07$ & $4.22 \pm 0.07$ \\
\hline trans-Cinnamic acid & 147 & $3.51 \pm 0.34$ & $6.13 \pm 2.97$ & $12.55 \pm 4.84$ & $9.72 \pm 0.54$ \\
\hline \multicolumn{6}{|l|}{ Stilbenes: } \\
\hline Resveratrol & $227(185,159)$ & $110.77 \pm 20.94$ & $93.67 \pm 16.47$ & $121.63 \pm 9.90$ & $75.73 \pm 9.89$ \\
\hline
\end{tabular}

Values are means of duplicate determination \pm S.D. nd: not detected. tr: trace.

ranged from $75.73 \mathrm{mg} \mathrm{RE} / \mathrm{kg}$ (MS) to $121.63 \mathrm{mg} \mathrm{RE} / \mathrm{kg}$ (SY) (Table 2). SY had a higher level of resveratrol than the three other varieties (Fig. 2e).

From the description above, the differences in anthocyanin contents among CS, ML, SY and MS were significant. However, there were no differences among CS, ML and MS for the flavonol, flavan-3-ol, phenolic acid and stilbene contents. This suggests that anthocyanins could be more useful for distinguishing grape varieties (Raúl et al., 2009). The phenolic composition of wines depends upon the grape variety and other factors that affect the berry development, such as soil, geographical location and weather conditions (Monagas et al., 2005). In the present study, under the same ecological conditions and cultivation management, the four grape cultivars displayed different phenol profiles, indicating that the biosynthesis of phenolic compounds depends largely on the genotype of the grape cultivar (Boss et al., 1996; Tian et al., 2008).

\section{Cluster analysis}

To better understand the phenolic characteristics of the four grape cultivars, cluster analysis using Ward's method was carried out on these identified phenolic compounds. As shown in Fig. 3, the four grape cultivars can be divided into two groups: CS and ML, and SY and MS. CS and ML were clustered within a short distance, indicating that CS and ML have similar phenolic compound profiles. A similar result for phenolic compounds was also observed between SY and MS. CS and ML showed great differences from SY and MS. This suggests that the profiles of the phenolic compounds in CS and ML were significantly different from those of SY and MS. 

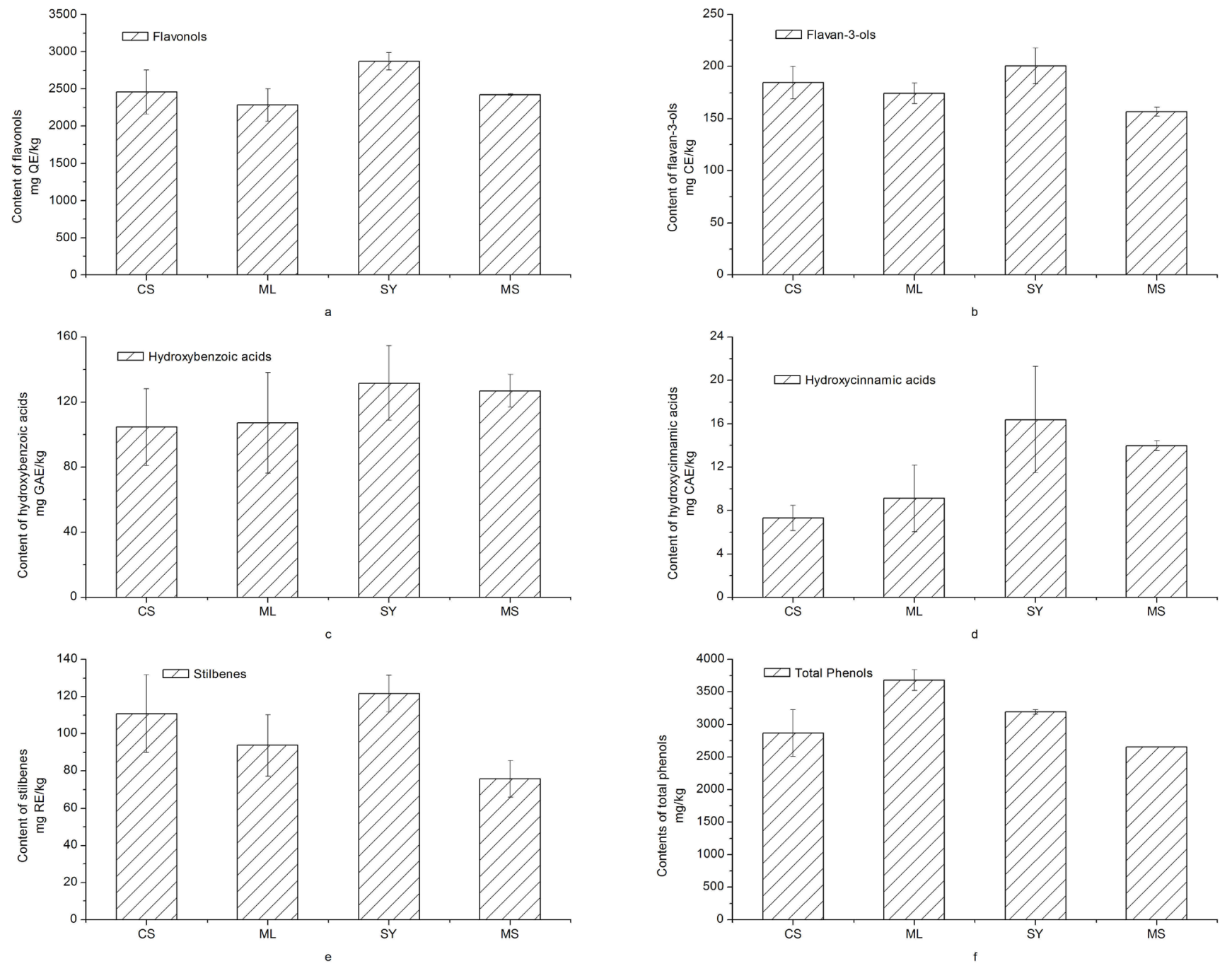

FIGURE 2

The total content of flavonols (a), flavan-3-ols (b), hydroxybenzoic acids (c), hydroxycinnamic acids (d), stilbenes (e) and total phenols (f) in the berry skins of the four grape cultivars. QE: quercetin; CE: catechin; GAE: gallic acid; CAE: caffeic acid; RE: resveratrol.

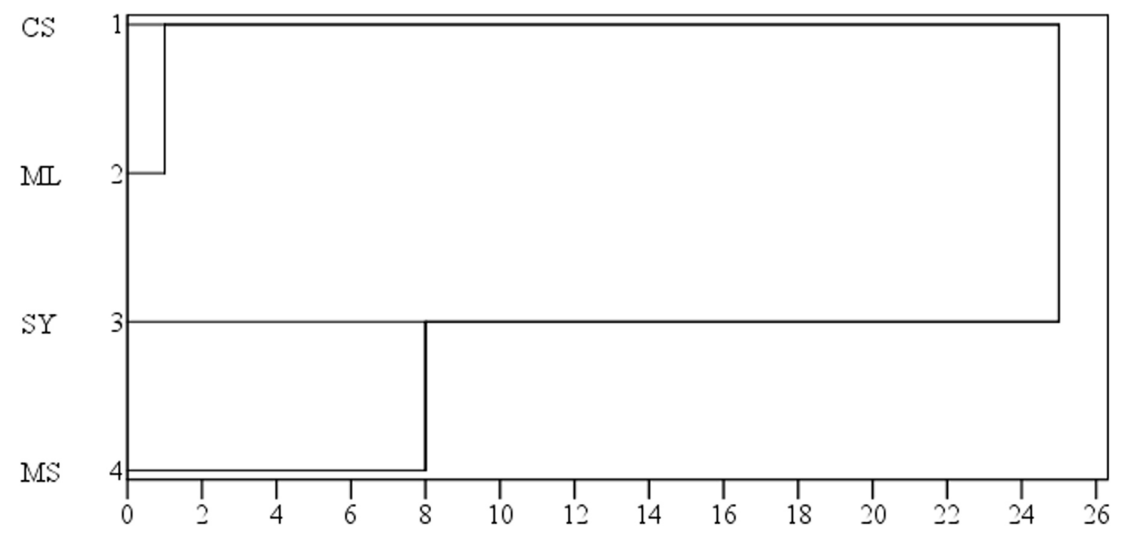

FIGURE 3

Cluster analysis of the four grape cultivars in Donghuayuan 


\section{CONCLUSIONS}

In summary, 21 different anthocyanins and 27 nonanthocyanin phenolic compounds were detected in CS, ML, SY and MS grape skins from grapevines at Donghuayuan town. The composition and content of anthocyanins showed significant differences between CS and ML, and SY and MS. It was shown that malvidin and its derivatives are the main anthocyanins present in the skin. SY and MS had a higher level of total anthocyanins and malvidin and its derivatives than CS and ML. The content of non-anthocyanins showed little difference among CS, ML and MS. Cluster analysis showed that CS and ML, and SY and MS had similar phenolic compound profiles.

The findings of this study are useful for optimising winemaking processes to produce a particular geographical indication of wines' origins, depending largely on a detailed analysis of their phenolic content. This approach is especially relevant to Shacheng (China), which has a warm, semiarid climate, yet is a traditional winemaking area of which the wine typicality and "terroir" characters need to be explored.

\section{LITERATURE CITED}

Boss, P.K., Davies, C. \& Robinson, S.P., 1996. Analysis of the expression of anthocyanin pathway genes in developing Vitis vinifera L. cv Shiraz grape berries and the implications for pathway regulation. Plant Physiol. 111, 1059-1066.

Boulton, R., 2001. The copigmentation of anthocyanins and its role in the color of red wine: A critical review. Am. J. Enol. Vitic. 52, 67-87.

Brescia, M.A., Caldarola, V., Giglio, A.D., Benedetti, D., Fanizzi, F.P. \& Sacco, A., 2002. Characterization of the geographical origin of Italian red wines based on traditional and nuclear magnetic resonance spectrometric determinations. Anal. Chim. Acta 458, 177-186.

Douglas, D., Cliff, M.A. \& Reynolds, A.G., 2001. Canadian terroir: Characterization of Riesling wines from the Niagara Peninsula. Food Res. Int. 34, 559-563.

Downey, M.O., Harvey, J.S. \& Robison, S.P., 2004. The effect of bunch shading on berry development on flavonoid accumulation in Shiraz grapes. Am. J. Enol. Vitic. 10, 55-73.

Fernandez, K., Kennedy, J.A. \& Agosin, E., 2007. Characterization of Vitis vinifera L. cv. Carmenere grape and wine proanthocyanidins. J. Agric. Food Chem. 55, 3675-3680.

Gómez-Alonso, S., García-Romero, E. \& Hermosín-Gutiérrez, I., 2007. HPLC analysis of diverse grape and wine phenolics using direct injection and multidetection by DAD and fluorescence. J. Food Compost. Anal. 20, 618-626.

Jensen, J.S., Demiray, S., Egebo, M. \& Meyer, A.S., 2008. Prediction of wine color attributes from the phenolic profiles of red grapes (Vitis vinifera). J. Agric. Food Chem. 56, 1105-1115.

Jin, Z.-M., He, J.-J., Bi, H.-Q., Cui, X.-Y. \& Duan, C.-Q., 2009. Phenolic compound profiles in berry skins from nine red wine grape cultivars in Northwest China. Molecules 14, 4922-4935.

Kallithraka, S., Mohdaly, A.A.A., Makris, D.P. \& Kefalas, P., 2005. Determination of major anthocyanin pigments in Hellenic native grape varieties (Vitis vinifera sp.): Association with antiradical activity. J. Food Compos. Anal. 18, 375-386.

Li, Z., Pan, Q.H. \& Jin, Z.M., 2011. Comparison on phenolic compounds in Vitis vinifera cv. Cabernet Sauvignon wines from five wine-growing regions in China. Food Chem. 125, 77-83.
Liang, Z., Wu, B., Fan, P., Yang, C., Duan, W., Zheng, X., Liu, C. \& Li, S., 2008. Anthocyanin composition and content in grape berry skin in Vitis germplasm. Food Chem. 111, 837-844.

Mateus, N., Machado, J.M. \& De Freitas, V., 2002. Development changes of anthocyanins in Vitis vinifera grapes grown in the Douro Valley and concentration in respective wines. J. Sci. Food Agric. 82, 1689-1695.

Mattivi, F., Guzzon, R., Vrhovsek, U., Stefanini, M. \& Velasco, R., 2006. Metabolite profiling of grape: Flavonols and anthocyanins. J. Agric. Food Chem. 54, 7692-7702.

Mazza, G., Fukumoto, L., Delaquis, P., Girard, B. \& Ewert, B., 1999. Anthocyanins, phenolics, and color of Cabernet Franc, Merlot, and Pinot Noir wines from British Columbia. J. Agric. Food Chem. 47, 4009-4017.

Monagas, M., Bartolome, B. \& Gomez-Cordoves, C., 2005. Updated knowledge about the presence of phenolic compounds in wine. Crit. Rev. Food Sci. 45, 85-118.

Mori, K., Sugaya, S. \& Gemma, H., 2005. Decreased anthocyanin biosynthesis in grape berries grown under elevated night temperature condition. Sci. Hortic. 105, 319-330.

Raúl, F., Liazid, G.A., Palma, M., Puertas, B., González-Barrio, R., GilIzquierdo, Á., García-Barroso, C. \& Cantos-Villar, E., 2009. Phenolic characterisation of red grapes autochthonous to Andalusia. Food Chem. 112, 949-955.

Revilla, E., García-Beneytez, E. \& Cabello, F., 2009. Anthocyanin fingerprint of clones of Tempranillo grapes and wines made with them. Aust. J. Grape Wine Res. 15, 70-78.

Revilla, E., Garcia-Beneytez, E., Cabello, F., Martín-Ortega, G. \& Ryan, J.M., 2001. Value of high-performance liquid chromatographic analysis of anthocyanins in differentiation of red grape cultivars and red wines made from them. J. Chromatogr. A. 915, 53-60.

Rodriguez, R.C., Aguilar, M.P. \& Gomez, A., 2006. Determination of some hydroxybenzoic acids and catechins in white wine samples by liquid chromatography with luminescence detection. J. Sep. Sci. 29, 2772-2779.

Salas, E., Fulcrand, H., Meudec, E. \& Cheynier, V., 2003. Reactions of anthocyanins and tannins in model solutions. J. Agric. Food Chem. 51, 7951-7961.

Spayd, S.E., Tarara, J.M., Mee, D.L. \& Ferguson, J.C., 2002. Separation of sunlight and temperature effects on the composition of Vitis vinifera cv. Merlot berries. Am. J. Enol. Vitic. 53, 171-182.

Sun, A.Y., Simonyi, A. \& Sun, G.Y., 2002. The "French paradox" and beyond: Neuroprotective effects of polyphenols. Free Radic. Biol. Med. 324, 314-318.

Sun, B.S., Santos, C.P.R., Leandro, M.C., De Freitas, V. \& Spranger, M.I., 2007. High-performance liquid chromatography/electrospray ionization mass spectrometric characterization of new products formed by the reaction between flavanols and malvidin-3-glucoside in the presence of acetaldehyde. Rapid Commun. Mass Sp. 21, 2227-2236.

Tarara, J.M., Lee, J., Spayd, S.E. \& Scagel, C.F., 2008. Berry temperature and solar radiation alter acylation, proportion, and concentration of anthocyanin in merlot grapes. Am. J. Enol. Vitic. 59, 235-247.

Tian, L., Pang, Y. \& Dixon, R.A., 2008. Biosynthesis and genetic engineering of proanthocyanidins and (iso)flavonoids. Phytochem. Rev. 7, 445-465.

Xing, R.R., He, F., Xiao, H.L., Duan, Ch.Q. \& Pan, Q.H., 2015. Accumulation pattern of flavonoids in Cabernet Sauvignon grapes grown in a low-latitude and high-altitude region. S. Afr. J. Enol. Vitic. 36(1), 32-43.

Zhang, M.X., Liu, C.H., Nan, H.J. \& Li, Z., 2015. Phenolic compound profiles in skins of white wine and table grape cultivars grown in the National Grape Germplasm Resource Nursery of China. S. Afr. J. Enol. Vitic. 36(1), 154-164. 